\section{Development of fatal systemic gas embolism during direct peroral cholangioscopy under carbon dioxide insufflation}
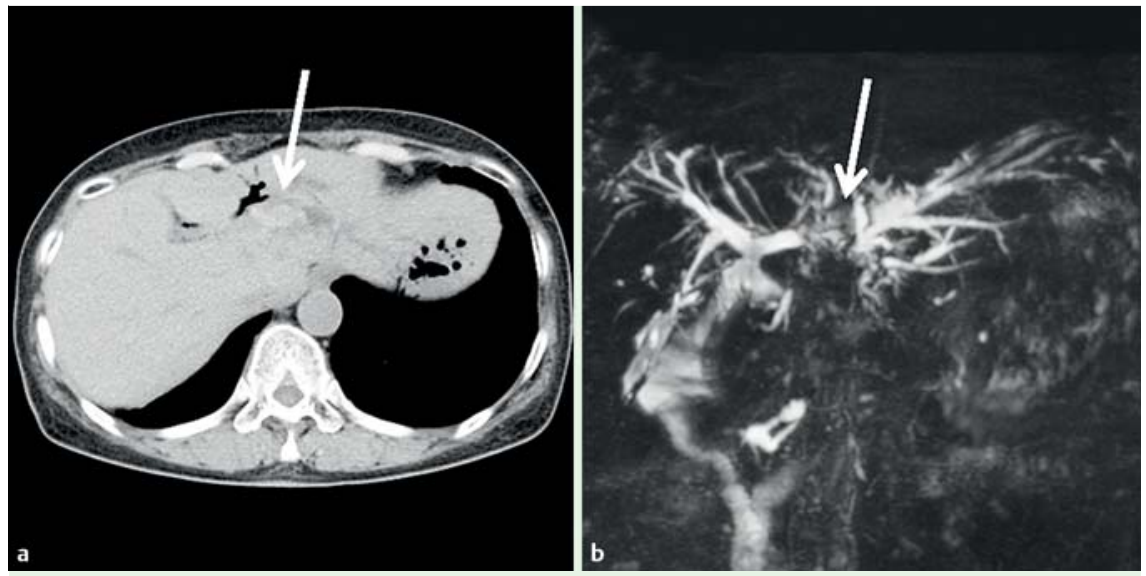

Fig. 1 Images of a hepatolith (white arrow), $20 \mathrm{~mm}$ in diameter, in the left intrahepatic bile duct on: a computed tomography (CT) scan; b magnetic resonance cholangiopancreatography (MRCP).
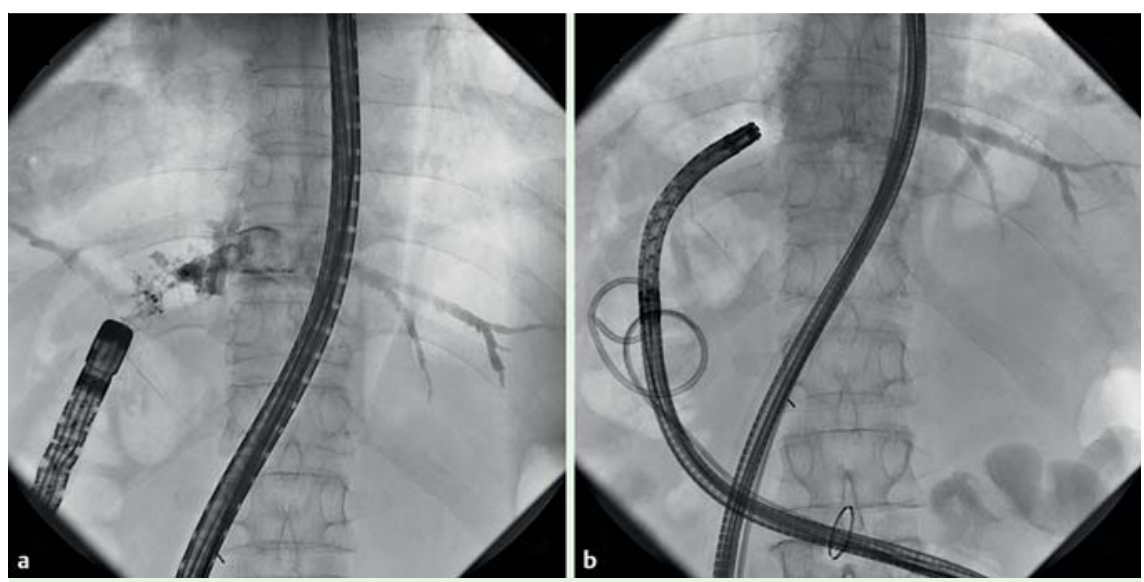

Fig. 2 Radiographic images showing: a an attempt to extract the stones through the double-balloon enteroscope (DBE) from the distal side of the anastomosis; $\mathbf{b}$ an ultraslim endoscope, which had replaced the DBE, advanced into the bile duct.

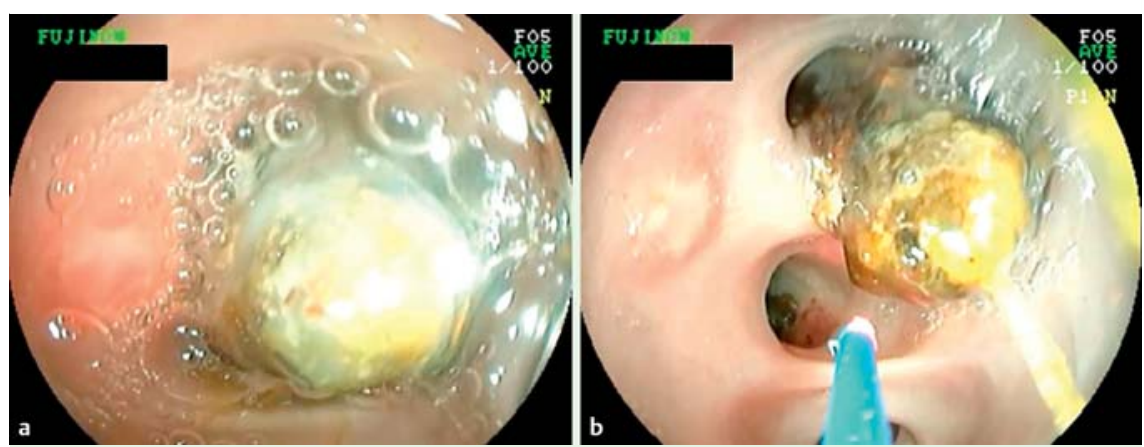

Fig. 3 Views via the ultraslim endoscope showing: a the hepatolith along with pus and mucus in the left intrahepatic bile duct; $\mathbf{b}$ the proximal bile duct beyond the hepatolith seen at the moment of lithotripsy using a Holmium:YAG laser after mucus and pus had been removed.
Direct peroral cholangioscopy (DPOCS) is a useful and effective technique for diagnosis and therapy of biliary tract disease [1-3]. No instances of a fatal systemic gas embolism developing during DPOCS under carbon dioxide $\left(\mathrm{CO}_{2}\right)$ insufflation have yet been reported.

A 68-year-old woman was admitted to our hospital with a complaint of hepatolithiasis ( $\bullet$ Fig. 1 ). She had undergone Roux-en-Y hepaticojejunostomy for choledochal cysts 34 years previously. We performed DPOCS using a short-type double-balloon enteroscope (DBE), an ultraslim endoscope, and an endoscopic $\mathrm{CO}_{2}$ regulator (EC-450BI5, EG-580NW, GW-1, respectively; Fujifilm Corp., Tokyo, Japan) while the patient was kept adequately sedated with midazolam.

We planned to perform lithotripsy of the hepatolith using a Holmium:YAG laser. After we had reached the anastomosis using the DBE, attempts to extract the stones through the DBE using balloon or basket catheters failed ( $\bullet$ Fig. $\mathbf{2 a}$ ). We therefore decided to perform DPOCS with an ultraslim endoscope passed through an overtube using a previously described method ( $\bullet$ Fig.2b) [3]. The balloon attached to the overtube remained inflated from the time that we reached the anastomosis until the end of the procedure.

We first confirmed the hepatolith was present $(\boldsymbol{G}$ Fig.3a). We then prepared the Holmium:YAG laser for lithotripsy for 5 minutes, while we aspirated pus and mucus discharged from the peripheral bile duct near the hepatolith. As we fractured the hepatolith with the Holmium:YAG laser ( $\boldsymbol{O} \mathbf{~ F i g . 3 b}$ ), the patient suddenly went into shock and had a cardiac arrest. Despite immediate cardiomegaly resuscitation and injection of flumazenil, she died. A computed tomography (CT) scan performed during resuscitation revealed multiple gas emboli in the systemic arteries and veins ( $\bullet$ Fig.4). Pathological examination later revealed hepatic abscesses, inflammation surrounding the hepatolith, intravascular gas, and systemic gas emboli [4]. There was no evidence of a patent foramen ovale [5]. The cause of death was systemic gas embolism. We believe aspiration of pus and mucus prior to lithotripsy may have opened a pre-existing biliovenous shunt. Endoscopists should take the possibility of fatal gas embolism into consideration during DPOCS even under $\mathrm{CO}_{2}$ insufflation. The extent of insufflation should be the absolute minimum required. 

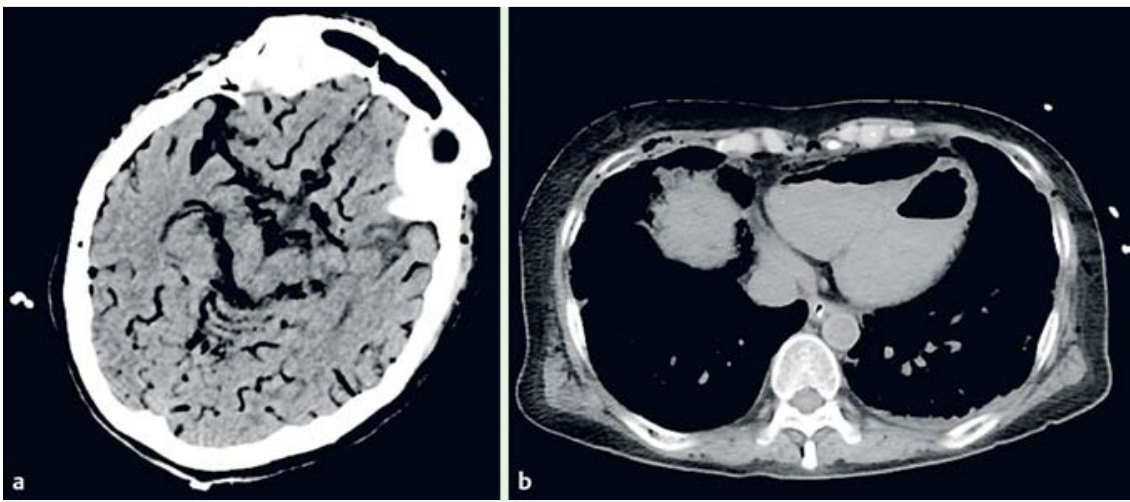

Fig. 4 Computed tomography (CT) scan during resuscitation showing multiple gas emboli in the systemic arteries and veins of: $\mathbf{a}$ the brain; $\mathbf{b}$ the heart.

Endoscopy_UCTN_Code_CPL_1AK_2AI

Competing interests: None

\section{Hiromu Kondo, Itaru Naitoh, Takahiro Nakazawa, Kazuki Hayashi, Yuji Nishi, Shuichiro Umemura, Takashi Joh}

Department of Gastroenterology and Metabolism, Nagoya City University Graduate School of Medical Sciences, Nagoya, Japan

\section{References}

1 Tringali A, Lemmers $A$, Meves $V$ et al. Intraductal biliopancreatic imaging: European Society of Gastrointestinal Endoscopy (ESGE) technology review. Endoscopy 2015; 47: 739-753

2 Moon JH, Ko BM, Choi HJ et al. Direct peroral cholangioscopy using an ultra-slim upper endoscope for the treatment of retained bile duct stones. Am J Gastroenterol 2009; 104: $2729-2733$

3 Itoi $T$, Sofuni A, Itokawa $F$ et al. Diagnostic and therapeutic peroral direct cholangioscopy in patients with altered GI anatomy (with videos). Gastrointest Endosc 2012; 75: $441-449$
4 Donepudi S, Chavalitdhamrong D, Pu L et al. Air embolism complicating gastrointestinal endoscopy: A systematic review. World J Gastrointest Endosc 2013; 5: 359-365

5 Finsterer J, Stollberger C, Bastovansky A. Cardiac and cerebral air embolism from endoscopic retrograde cholangio-pancreatography. Eur J Gastroenterol Hepatol 2010; 22: $1157-1162$

Bibliography

DOI http://dx.doi.org/

10.1055/s-0042-109056

Endoscopy 2016; 48: E215-E216

(c) Georg Thieme Verlag KG

Stuttgart · New York

ISSN 0013-726X

\section{Corresponding author}

Itaru Naitoh, MD

Department of Gastroenterology and Metabolism Nagoya City University Graduate School of Medical Sciences

1 Kawasumi, Mizuho-cho, Mizuho-ku Nagoya 467-8601

Japan

Fax: +81-52-8520952

inaito@med.nagoya-cu.ac.jp 